\title{
Dispositionality, categoricity, and where to find them
}

\section{Lorenzo Azzano ${ }^{1}$}

Received: 4 February 2020 / Accepted: 14 October 2020 / Published online: 18 November 2020 (c) The Author(s) 2020

\begin{abstract}
Discussions about dispositional and categorical properties have become commonplace in metaphysics. Unfortunately, dispositionality and categoricity are disputed notions: usual characterizations are piecemeal and not widely applicable, thus threatening to make agreements and disagreements on the matter merely verbal—and also making it arduous to map a logical space of positions about dispositional and categorical properties in which all parties can comfortably fit. This paper offers a prescription for this important difficulty, or at least an inkling thereof. This will be achieved by comparing pairs of positions and exploring their background metaphysics to discover where alleged agreements and disagreements concerning dispositionality and categoricity really lie; more specifically, the Pure Powers view (according to which properties are just dispositional) and the Powerful Qualities view (according to which properties are also categorical) will be under scrutiny. Over this background, the prescription functions by isolating a successful identity-based characterization of categoricity, while abandoning the correspondent identity-based characterization of dispositionality. On the contrary, according to this prescription a property is dispositional if and only if it is solely in virtue of possessing that property that its bearer is assigned a certain dispositional profile. A crucial consequence of this prescription is that, while supporters of the Pure Powers view often characterize their position as an essentialist one, the dispositionality of properties needn't always be a matter of essence.
\end{abstract}

Keywords Dispositional properties · Categorical properties $\cdot$ Pure powers $\cdot$ Powerful qualities $\cdot$ Categoricalism $\cdot$ Grounding

\section{Introduction}

Discussions about whether properties are dispositional or categorical have become commonplace in recent metaphysics and philosophy of science; yet there is no ortho-

Lorenzo Azzano

lorenzo.azzano@unibg.it; lorenzo.az@hotmail.it

1 Department of Letters, Philosophy, Communication, University of Bergamo, Via Pignolo 123, CAP 24121 Bergamo, Italy 
dox, or even widely accepted characterization of a dispositional property as opposed to a categorical one. ${ }^{1}$ This isn't a new difficulty, either: early discussions on the so-called "mark of the dispositional", conducted either through conditional entailment or the association with intentionality, reveal the struggle the first contemporary supporters of dispositional properties had to go through to narrow down their subject of enquiry; as for the categorical properties, more than twenty years ago Mumford (1998: p. 75) writes that.

it is quite difficult to find, anywhere in the literature, a specification of what exactly is intended by 'categorical property' and it is a symptomatic of the vagueness of the distinction accepted thus far that a number of different things are usually put forward as examples of the categorical.

Paradigmatic examples abound: e.g. elasticity for dispositionality, and triangularity for categoricity. But why is elasticity dispositional? Why is triangularity categorical? The brief debate in Mellor (1974, 1982) and Prior (1982) on "counting corners correctly" offered perhaps the first instance in which the dispositional/categorical divide (in this case, subjunctive conditional entailment) did not respect a prior demarcation; and Mellor's conclusion - that insofar as all property ascriptions entail conditionals, all properties are dispositional—could be reversed so as to cast some doubts on the idea that conditional entailment is indeed the way to go. ${ }^{2}$

This difficulty has not been solved. Quite the contrary: the chaos is now so rampant that even amongst friends of powers there are those who consider dispositionality and categoricity mutually exclusive categories (viz., dispositional properties cannot be categorical, and vice versa), and those who don't. In fact, traditionally mutually exclusive characterizations are now challenged by those who take properties to be both dispositional and categorical. This, of course, creates a danger: the multiplicity and heterogeneity of such characterizations is such that, whenever two parties agree (or disagree) as to whether properties are dispositional (or categorical), it is possible that their characterizations are not lining up, and they might be talking past each other. This ultimately makes it very difficult to map a logical space of positions in which all parties can comfortably fit.

Yet there are, or so I argue, good news: there is a characterization of dispositionality and categoricity which is acceptable for all parties involved, albeit not one that has always been clearly put forward by such parties. This conclusion will be reached through a method by comparison: by comparing various positions, I will explore their background metaphysics to discover where their alleged agreements and disagreements concerning dispositionality and categoricity really lie. This solution is mostly prescriptive in character, in the sense that I will deem certain characterizations

\footnotetext{
${ }^{1}$ Two clarifications are needed. First: for the sake of this paper, I will speak of dispositionality and categoricity as applied to properties; thus, the non-trivial truth of sentences concerning dispositionality and categoricity depends on the existence of properties. Secondly: as many others discussing these topics (e.g., Bird 2007a), I am primarily concerned with fundamental, or perfectly natural properties; this restriction will be important later on (Sect.6.2 ). For a more liberal and unconventional take on dispositionality, see McKitrick (2018).

2 Different conclusions are reached, e.g., in Mumford (1998: pp. 67-819), Ellis (2001: pp. 121-127), and Bird (2003, 2009).
} 
unsatisfactory (according to certain criteria), and prescribe others instead, to ensure proper communication between two parties and ultimately prescribe a clearer terrain of engagement for everyone. ${ }^{3}$

This will be a long ride: in the next Section, I will introduce this method by comparison, including the criteria for a satisfactory characterization of dispositionality and categoricity; I will also introduce the positions discussed in the remainder of the paper: the Pure Powers view and the Powerful Qualities view. These allegedly disagree on whether properties are just dispositional, or also categorical. But do they really? In Sect. 3 the characterizations of dispositionality and categoricity put forward by supporters of the Powerful Qualities view will be analyzed, and many shortcomings will be found; and similarly for the characterizations put forward by supporters of the Pure Powers view in Sect. 4; these are mostly essence-or identity-based characterizations, and whether they satisfy the required criteria will take up the entirety of Sect. 5. The upshot of these three sections is that, shockingly, it is quite hard to find common ground.

In Sect. 6 I will submit my prescription and present its virtues. Perhaps the most crucial consequence of this prescription is that, while supporters of the Pure Powers view often characterize their position as one according to which properties have a dispositional essence, this needn't be a claim shared by all friends of powers: dispositionality need not be a matter of essence. In Sect. 7 some further features and potential objections to my prescription are discussed-which may offer future directions of enquiry for friends of powers. Finally, Sect. 8 will offer some conclusions.

\section{Method of inquiry}

A twofold question of method immediately arises. Firstly: how to proceed in my quest for dispositionality and categoricity? It would be too long to just scout the literature for various characterizations of dispositional and categorical properties, hunting down analyses, so to speak, until we found a satisfactory one. And secondly: what would make for a satisfactory characterization anyway?

The method I put forward is one by way of comparison. If, for the sake of the argument, we exclude dualist positions, viz. positions according to which we find two distinct kinds of properties instantiated in the world, we end up with three families of positions: according to the Dispositionalist family, there only are dispositional properties, which are not categorical; according to the Categoricalist family, there only are categorical properties, which are not dispositional; finally, according to the Mixed family, there are properties which are both dispositional and categorical. With "friend of powers" I mean anyone who holds any position in the Dispositionalist or Mixed family; with "foe of powers" I mean anyone who holds any position in the Categoricalist family.

\footnotetext{
3 Relatedly, I do not require anything as strong as a genuine definition -a full story about what it is to be a dispositional or categorical property; I would rather settle for adequate criteria, namely necessary and sufficient conditions for something being a dispositional or a categorical property. This is what I will call a "characterization".
} 
Any position in the Dispositionalist family is supposed to be similar to any position in the Mixed family in one respect (because according to both, there are dispositional properties), and is supposed to be dissimilar to it in another respect (because according to one, but not the other, there are categorical properties); and something similar goes for any two positions in the Mixed and Categoricalist family (they are similar in one respect: there are categorical properties; but dissimilar in another: according to one, but not the other, there are dispositional properties). Thus, e.g., a supporter of a position in the Dispositionalist family will agree with a supporter of a position in the Mixed family on the existence of dispositional properties, but disagree with them on the existence of categorical ones: such agreements and disagreements on matters of dispositionality and categoricity are based on the alleged similarities and dissimilarities between the respective positions. We can thus compare the background metaphysics of any two pairs of positions in the three families (more specifically, their purported characterizations of dispositionality and categoricity) to check whether their similarities and dissimilarities in those respect are genuine or not. If they are not, such agreements and disagreements are not genuine either-but merely verbal.

Thus, we can evaluate characterizations of dispositionality and categoricity by checking whether they ensure alleged agreements and disagreements between pairs of positions. More specifically, a characterization of dispositionality or categoricity must satisfy two crucial criteria: it must be comprehensive and it must be divisive. Firstly, it must be comprehensive in the sense that it must be applicable to the properties of any two positions which are similar in the relevant respect: in a comparison between a position in the Dispositionalist family and another in the Mixed family, a characterization of dispositionality needs to be applicable to properties of both positions, so as to ensure genuine similarity between them. Secondly, it must be divisive in the sense that it must not be applicable to properties of any two positions which are dissimilar in the relevant respect: in a comparison between a position in the Dispositionalist family and another in the Mixed family, a characterization of categoricity has to be applicable to properties of the second but not the first position, so as to ensure genuine dissimilarity between them.

In short, comprehensiveness ensures genuine similarity between positions, while divisiveness ensures genuine dissimilarity between positions: this is our rough guide in our quest for dispositionality and categoricity.

This method is not admittedly a very strict one: most importantly, there still is an amount in liberty in the choice of which positions to compare. This might be understood as a problem of scope for comprehensiveness and divisiveness; roughly put, the wider we cast our net, the less orthodox positions we are bound to encompass: but where to stop? This problem becomes acute in the case of the somewhat heretical positions in the Mixed Family, for which properties are both dispositional and categorical; e.g., Armstrong (2005: p. 315) famously considered such positions (or at least one of them) "totally incredible", and involving a "category mistake"; someone sharing this opinion wouldn't be too worried if we weren't able to formulate a comprehensive characterization of dispositionality, so as to allow the supporter of this "totally incredible" position to fall within the ranks of the friends of powers.

This is a legitimate worry, but two things must be said. Firstly: there is an ineliminable element of vagueness at play here, that cannot possibly be dispelled: I will 
begin by considering positions in the three families as they have been described and accepted by the ensuing literature; of course this may inject an arbitrary element in the discussion, but a (partly) arbitrary starting point is better than no starting point. Secondly, I am more worried about casting a scope which is too narrow, rather than too wide; back to the Armstrong case, in our current situation we shouldn't assume anything about dispositionality nor categoricity, not even that dispositional properties are non-categorical. To do the opposite would have no effect, except occluding logical space: for now, the burden of proof squarely lies at the feet of those who want to start with mutually exclusive categories.

Let us now introduce the positions to be discussed: I will focus on the comparison between two popular positions amongst friends of powers, the Pure Powers view (for brevity, PP), and the Powerful Qualities view (PQ) - supporters of PP I will call "PPers", while supporters of PQ I will call "PQers". According to PP, properties are exhaustively dispositional; there are no categorical properties; according to PQ properties are both dispositional and categorical. Thus PP belongs in the Dispositionalist family, whereas PQ belongs in the Mixed family. PP is supposed to be similar to PQ in one respect (they agree that there are dispositional properties), but dissimilar in another respect (they disagree on the existence of categorical properties); similarly, PQ is supposed to be similar to Categoricalism in one respect (they agree that there are categorical properties), but dissimilar in another respect (they disagree on the existence of dispositional properties). Following the criteria above, I will explore which characterizations of dispositionality and categoricity ensure genuine similarities and dissimilarities between them, thus laying the foundations for a correct reconstruction of the dialectic between the two positions (and Categoricalism).

I will begin with the characterizations offered by the PQer, then move on to those of the PPer. Finally I will offer my prescription.

\section{$3 \mathrm{PQ}$ on dispositionality and categoricity}

According to PQ, properties are both dispositional and categorical (or qualitative). ${ }^{4}$ PQ has its fair share of supporters: Martin (1993, 1997, 2008), Martin and Heil (1999), Heil (2003), Strawson (2008), Engelhardt (2010), Schroer (2010, 2013), Jacobs (2011), Ingthorsson (2013), Taylor (2013, 2018), Williams (2019), and Giannotti (Forthcoming). Thus, there understandably are different variants of the position on the market. I will highlight such differences when needed; for now, it is only important to point out that PQ's claim that properties are both dispositional and categorical should be understood as a metaphysically substantial claim. What does that mean? PQ has a cousin in the so-called Neutral Monist view (NM, as in Mumford 1998) according to which properties are both dispositional and categorical in the weaker sense that both dispositional and categorical descriptions or conceptualizations apply to them; but properly speaking, according to NM, it is not properties-but predicates or concepts_-, to be dispositional or categorical. PQ is not always clearly distinguished from

\footnotetext{
4 In the current debate, the terms "categorical" and "qualitative" are being used almost synonymously; I will, for the most part, adhere to this custom, but in Sect. 7.4 I will examine this conflation under a more critical light.
} 
NM. ${ }^{5}$ Yet in our context, the distinction matters: a supporter of NM would have no problem admitting that agreements and disagreements on matters of dispositionality and categoricity are merely verbal, or, at least, they do not pick up any genuine difference in one's background metaphysics. So this is something we must keep an eye on, when discussing PQ: for we intend investigating dispositionality and categoricity as genuine metaphysical categories. ${ }^{6}$

Let us begin. Naturally, to the extent in which dispositionality and categoricity were traditionally mutually exclusive categories, $\mathrm{PQ}$ is already a very peculiar position; and it already imposes some restrictions on acceptable characterizations of the two notions: in fact, my criteria exclude any characterization states or entails that dispositional properties are not categorical, or vice versa.

\subsection{PQ on categoricity}

Let us start with the categoricity, or qualitativity, of powerful qualities. It is often claimed that categorical properties are actual, non-conditional features of their bearers; supporters of PQ often agree with this characterization; for one Heil (2012: p. 59) claims that.

qualities are categorical; qualities are here and now, actual, not merely potential, features of the objects of which they are qualities.

Sometimes the notion of categoricity is imbued with a little more content: challenging the long-standing philosophical tradition that distinguishes "categorical properties from dispositional properties $[\ldots]$ on the grounds that categorical properties are intrinsic and dispositional properties are not", Heil (2003: pp. 78-79) characterizes the qualitativity of powerful qualities by submitting that.

I shall use 'qualitative' to designate intrinsic qualitative properties of objects, properties often classified as 'categorical'.

Actuality and intrinsicality do not exhaust the usual range of connotations concerning qualitativity. Ingthorsson (2013: p. 58) claims that "the notion of pure quality is a part and parcel of many mutually exclusive ontological views", but he goes on to propose that there is a "proto-idea" of quality, viz. a property that is "simple, first-order, independent, intrinsic, monadic, objective, actual or occurrent, and determinate." Furthermore, PQers occasionally stress out that powerful qualities are qualitative, in the sense that they are a way the object qualitatively is, or, in other words, they "contribute to the overall makeup of how an object is now" (Taylor 2013: p. 94).

This loose collection of characterizations may very well satisfy comprehensiveness: they are applicable to the properties of the Categoricalist (with which the PQer agrees about the existence of categorical properties). Unfortunately, they fail the test of divisiveness, and thus fail to properly distinguish powerful qualities from pure

\footnotetext{
5 Ingthorsson (2013: p. 56) characterizes PQ as the position according to which "properties either are both qualities and powers, or neither [...] The difference between 'both' or 'neither' is negligible.".

6 This is not to say that I reject NM. Like dualist positions, I am consciously excluding NM to reconstruct a smaller portion of the logical space.
} 
powers. This is no news, of course; it is old news that if a categorical property is an actual property, then dispositional properties are categorical: "[d]isposition ascriptions are categorical in the sense that to say that something has a dispositional property is to say that something as a property actually" (Mumford 1998: p. 37). To claim that dispositional properties are actual is a prerogative of all friends of powers, and the correct answer to the admittedly question-begging "always packing, never travelling" actuality regress argument (as in Mumford 2004: pp. 174-175).

In fact, pure powers count as categorical according to all of the characterizations above; using Bird (2007a) as a reference point for PP, one can see that pure powers are in fact first-order (Bird 2007a: p. 140), intrinsic (Bird 2007a: p. 166), actual (Bird 2007a: p. 105), and determinate (Bird 2007a: p. 136). The PPer will also agree that properties contribute to the way in which an object qualitatively is, or that they are qualitative in the sense that they are involved in a philosophically meaningful restriction in the second-order quantification in indiscernibility principles_-viz., they are the non-haecceitistic genuine properties that count when qualitative indistinguishability is on the table.

Thus, no such characterization of categoricity can ensure genuine dissimilarity between PP and PQ, and ensure a non-merely-verbal disagreement as to whether properties are categorical. A similar point has been recently made in Taylor (2018: pp. 1425-1426), ultimately leading him to the conclusion that PP and PQ are not really distinct positions (as we will see, things are more complicated than that).

The categoricity of properties, in this sense, seems to be posited to ensure that these properties are bona fide properties, rather than, say, counterfactual assertions about their subjects. E.g., according to Ellis and Lierse (1994: p. 24), the job of categorical properties is to "characterize", namely: "[i]f two things have a categorical property in common, then they could be the same or similar in this respect"; yet to account for similarities appears to be a job of genuine properties tout court, rather than any subset thereof. So understood, the PQer's claim that dispositional properties are categorical deflates into the claim that dispositional properties are genuine properties-which appears to be the signature claim of any friend of powers, rather than the PQer specifically.

\subsection{PQ on dispositionality}

The association between categoricity and actuality has sometimes been accompanied by a correspondent association between dispositionality and potency; and PQ has often been characterized as the claim that powerful qualities are a mixed bag of act and potency. For Martin (1997: p. 215):

[t]he dream of either a purely qualitative, non-dispositional or a purely dispositional account of properties is philosophical fantasy. No property is in 'pure act', free of all unfulfilled potency unless it is a property of God (or perhaps the number 2).

Relatedly, sometimes PQers characterize their position by claiming that, as in Heil (2003: p. 11), 
[p]roperties contribute in distinctive ways to the dispositionalities and to the qualities of their possessors. This might be put by saying that a property is a quality and is a power.

This at least suggests that a property is dispositional to the extent that it contributes to the modal or dispositional profile of its bearer, viz. it is part of an explanation as to why the bearer would behave in a certain way in certain counterfactual situations, or it is part of an explanation as to why the bearer is disposed to so behave.

Such a characterization of dispositionality is, again, comprehensive but not divisive. It is comprehensive since the properties of the PPer, qua dispositional, presumably can be characterized along the lines sketched above; yet it is not divisive, since the properties of the Categoricalist also can. If the dispositionality of a property simply amounts to the fact that the property contributes to the modal or dispositional profile of its bearer, so much so that the only "non-dispositional" properties Martin can muster to mention are the properties of God or the number 2, then even the properties of the Categoricalist would count as dispositional too. After all, paradigmatic Categoricalist David Armstrong surely wouldn't deny that properties contribute to determine the modal or dispositional profile of its bearer; for one, Armstrong (1997: p. 69) claims that we think of properties "as bestowing powers upon the particulars that have them. A property that bestows no power will not be easy to detect!". A similar line of thought is offered by Heil (2003: p. 118) in defence of PQ, as he submits that "[a] pure quality, a property altogether lacking in dispositionality, would be undetectable and would, in one obvious sense, make no difference to its possessor." Does Armstrong unknowingly score a point for PQ? Unlikely; the PQer has merely selected a non-divisive criterion for dispositionality.

Luckily, there are more incisive characterizations of dispositionality. Heil (2003: p. 79, emphasis mine) proposes:

I shall use 'dispositional' to designate properties that bestow powers on their possessors in the following sense: it is solely by virtue of possessing a given dispositional property that an object possesses a given power.

Similarly, Taylor (2013: p. 94, emphasis mine) submits that.

a dispositional property is any property to which it is essential that it conveys upon the object that instantiates it the power to behave in a certain way given certain stimuli.

This suggests the more robust claim that a property is not merely dispositional to the extent in which it contributes to the modal or dispositional profile of the property (all properties do that!)—but to the extent in which it does it exclusively, or essentially. Relatedly, some PQers may believe that such a special relation between properties and dispositional profiles obtains because "[d]ispositional properties, if there are any, have their powers "built in"” (Heil 2003: p. 79). Albeit not often elaborated, this suggestion about the character of dispositionality is quite common amongst PQers; in what follows, I will refer to this loose collection of characterizations with the umbrella term Privileged Contributory Dispositionality. In fact, some characterization along these lines might prove to be a better candidate for our role of dispositionality. 
But to see which one, and why, we need to discuss dispositionality and categoricity as understood by the PPer.

\section{PP on dispositionality and categoricity}

According to PP there are only properties which are exclusively dispositional; unlike PQers, PPers are somewhat harder to find. Beside the already mentioned Bird (2007a, 2007b), we can count Shoemaker (1980) and Mumford (2004); perhaps, as we will later see, Hawthorne (2001) can also join in the ranks.

The PPer's characterizations of dispositionality and categoricity are very different from those that we have seen so far, which makes it challenging to find common ground. We cannot, as in the previous Section, discuss separately the two notions, as for the PQer they much more intimately related.

Before beginning, it might be interesting to notice that PPers occasionally understand the dangers of non-divisive characterizations of categoricity (e.g., Bird 2007a: p. 66). That said, they often make mistakes on their own: Bird (ibid.) prefaces its discussion on categoricity by claiming that "[w]hat we mean by 'categorical' must be understood in negative terms". Here Bird is following here a traditional divide set up by negation, as in Mumford (1998: p. 75): “[m]ost obviously, but least committally, a categorical property is any property which is not a dispositional property".

Some additional details are offered, however, which are worth discussing. Bird, and possibly other PPers, often spell out the dispositional and categorical status of properties in relation to quidditism. There is no single position which warrants the label "quidditism"; that said, all quidditistic positions fall in two broad variants, Modal Quidditism and Identity-Based Quidditism — as in Smith (2016). Both have been deployed in the characterization of dispositionality and categoricity by the PPer. Let us first introduce both variants of quidditism and their relation; then, in the following subsection, I will examine their deployment from the PPer.

\subsection{Two variants of quidditism}

According to Modal Quidditism, as in Bird (2007a: p. 71), properties are only contingently associated with a certain dispositional profile; these properties I will call "modal quiddities". Modal Quidditism reflects the peculiarly neo-Humean view on properties according to which, insofar as we accept trans-world identity for properties, the same property can elicit wildly different behaviors in its bearer from one possible world to another. This is the position dubbed by Smith (2016) "R-quidditism", viz. a position about the lack of restrictions in the recombination of properties and dispositional profiles across worlds. This allows for "total swaps", viz. a pair of distinct possible worlds $\mathrm{w}_{1}$ and $\mathrm{w}_{2}$, indistinguishable but for the fact that modal quiddities $\mathrm{p}_{1}$ in $\mathrm{w}_{1}$ and $\mathrm{p}_{2}$ in $\mathrm{w}_{2}$ have swapped places, in the sense that everything instantiating $\mathrm{p}_{1}$ in $\mathrm{w}_{1}$ instantiates $\mathrm{p}_{2}$ in $\mathrm{w}_{2}$ (and vice versa), and furthermore $\mathrm{p}_{1}$ and $\mathrm{p}_{2}$ have completely swapped their dispositional profiles. Thus, Modal Quidditism can be sum- 
marized in the claim that " $[\mathrm{t}]$ wo different possibilities can differ just by a permutation of fundamental properties" (Lewis 2009: p. 209).

A second variant is Identity-Based Quidditism, according to which properties have a primitive identity, which does not depend on any feature they may possess, as in Black (2000: p. 92) and (Bird 2007a: p. 72); this is the position dubbed by Smith (2016) "I-quidditism", viz. a position about the individuation of properties. This too, unfortunately, is too coarse-grained to individuate a specific position: firstly, that properties have a primitive identity may be understood as the claim that properties are primitively identified or individuated, in the sense that there are primitive identity or individuality facts involving them (viz., primitive facts about properties being identical, or primitive facts about which property is which). This claim about the identity and individuation of properties occasionally translates into a claim about their nature: guided by Lewis' (1986: p. 205) claim that "[t]here isn't much to the intrinsic nature of a universal", one could insist that quidditistic properties are those whose only nature is to be primitively identified or individuated, and, as such, lack any substantial nature. All that there is to them is their numerical identity and difference, as in Armstrong (1997: p. 168, 2004: p. 146), and (Bird 2007a: p. 3); such properties are occasionally called "thin quiddities". Yet it is also viable to claim that, although properties are primitively identified or individuated, they are in fact not so thin, and display some further intrinsic character (this subtle distinction, as far as I know, is only explicitly recognized in Smith 2016). Another position yet is one according to which properties do have a further intrinsic character (or, as it is sometimes called, a "thick quiddity"), which fixes their identity: according to this position, such identity or individuality facts are therefore not brute - the thick quiddity is. Finally, as in Locke (2012) we may endorse "extravagant quidditism" and reject the (often implicit) assumption that the property and the thick quiddity are one and the same, to reach the somewhat baroque position according to which there is an item called quiddity (distinct but somehow related to the property) which is what ultimately fixes its identity. In conclusion, Identity-Based Quidditism is not so much as a definite position, but rather a family thereof. ${ }^{7}$

It will be worthwhile to briefly explore the relation between Modal Quidditism and Identity-Based Quidditism. I agree with Smith (2016) that Identity-Based Quidditism doesn't entail Modal Quidditism: that properties have a primitive identity, in whichever way one wants to spell it, doesn't entail that they only contingently have the dispositional or nomic profiles they have, for the supporter of Identity-Based Quidditism may posit any number of restrictions in the recombination of properties and such profiles. After all, one may claim that a property possesses a primitive identity, in any of the senses detailed above, while at the same time being necessarily associated to a certain dispositional profile, necessary but not sufficient for its individuation. Thus a quiddistic property according to Identity-Based Quidditism may not be a modal quiddity. This point will play a crucial role.

As for the other direction, unlike Smith (2016) I submit that Modal Quidditism does not entail Identity-Based Quidditism. Modal Quidditism, as the claim that no dispositional, causal, or nomic profile of a property is necessary to it, entails that no

\footnotetext{
7 For the interested reader, the tripartite distinction built by Smith (2016) upon the framework of Locke (2012) offers an excellent entry-point on the subject.
} 
such profile can individuate it. Now, of course this is compatible with many instances of Identity-Based Quidditism. Smith (2016: p. 240) tries to reach the stronger conclusion that Modal Quidditism entails (some version of) Identity-Based Quidditism-in her formulation, R-quidditism entails I-quidditism-, by claiming that.

if R-quidditism is true and there is no restriction on how fundamental properties can recombine $[. .$.$] then fundamental properties cannot be individuated on the$ basis of their nomological roles and (something akin to) I-quidditism must be true.

But I take there to be a gap in her argument: roughly put, that the property cannot be individuated by any dispositional or nomic profile doesn't entail that it is primitively individuated. Something else might be doing the individuating. For example, according to Lowe (2010: 20),

[sphericity] has a distinct purely geometrical 'real' definition which expresses its essence and therefore serves as a ground of its transworld identity-without any implication that the latter is 'primitive' and 'mysterious'.

We thus arrive at Qualitative Identity, a more general claim than Identity-Based Quidditism. One may want to introduce Qualitative Identity as the claim that properties have their identity fixed either qualitatively or primitively. But that claim is loaded with an unfortunate circularity, since qualitativity is exactly what we are trying to characterize. Thus I will content myself with a negative, weaker, claim: according to Qualitative Identity, properties do not have their identity fixed by any dispositional or nomic profile, but in some other way -whether primitively or not. ${ }^{8}$

Summarizing: Modal Quidditism does not entail Identity-Based Quidditism, and neither the other way round. Yet they are both entailed by Qualitative Identity, which is thus a more general position (whether it is really a version of "quidditism", is a verbal dispute I am not particularly interested in pursuing).

\subsection{PP and modal quidditism}

Both Modal Quidditism and Identity-Based Quidditism have been intermittently used by PPers to characterize categoricity and dispositionality, but they are not clearly distinguished. ${ }^{9}$ E.g., Bird (2007a: p. 44) claims that "[c]ategorical properties [...] do not have their dispositional characters modally fixed", but shortly after he adds that "categorical properties have primitive identity".

Let us start from Modal Quidditism, which has been deployed by PPers in multiple occasions (most clearly, in Bird 2007a: pp. 66-67). This characterization is widely shared by many Categoricalists, who often endorse a properly neo-Humean contingency about laws of nature, so that which dispositional profile accompanies a property at some world depends on which laws happen to hold at that world. Categorical properties qua modal quiddities are often accompanied by a correspondent modal

\footnotetext{
8 As we will see in Sect.5, this negative formulation of Qualitative Identity is not without its problems. However, in the end it will be vindicated.

9 As far as I know, no PPer explicitly frames categorical properties along the lines of Qualitative Identity.
} 
characterization of dispositionality according to which dispositional properties are those that are necessarily, rather than contingently associated with their dispositional profile (most recently, Bird 2016: p. 6); this makes the divide between the categorical and the dispositional a modal one, a conclusion to which Bird has always been sympathetic (e.g., Bird 2007a: pp. 66-67, Bird 2016).

Such characterizations, however, will not do. Firstly, they are mutually exclusive-thus, unfairly ruling out PQ as a viable position. Furthermore, there are even more substantial difficulties when our two criteria are considered. For the characterization of categoricity is neither comprehensive nor divisive, while the characterization of dispositionality, while comprehensive, is not divisive.

Let us start with categoricity. If the characterization of categorical properties qua modal quiddities is to be comprehensive, it has to be applicable to the properties of all pairs of positions which agree on the existence of categorical properties. While this characterization may prima facie be applicable to the properties of the Categoricalist, it is not applicable to the properties of the PQer, even though the two agree on the existence of categorical properties. Crucially, powerful qualities are not modal quiddities: according to PQ, properties necessarily have the dispositional profiles that they have; furthermore, although PQers do not usually frame their positions in terms of laws of nature, they still take them to be a necessary matter-thus ensuring that properties have fixed dispositional profiles across the modal space (e.g., Heil 2003: pp. 92-95, 2005: pp. 345-346).

Additionally, the characterization of categorical properties qua modal quiddities is not divisive. This requires some elaboration. Framing categoricity through the lenses of Modal Quidditism makes the categoricity of properties a matter of modal recombination, or perhaps of modal status of laws of nature; yet if that were the case, no Categoricalist would be in a position to argue that laws of nature are metaphysically necessary. At times, this consequence seems to be endorsed by PPers; e.g., Bird (2007a: p. 4) proposes a table of positions according to which Categoricalists are forced to the claim that laws are contingent. But this is incorrect: it is important to keep in mind that Categoricalism, as the claim that all properties are categorical, is distinct from the claim that laws of nature are contingent. Separating these occasionally conflated claims we obtain, as in Azzano (2019: p. 347), a Categoricalist position provided with metaphysically necessary laws of nature; according to such a position, thus, while there are categorical properties, there are no modal quiddities. Let us call it NC, short for Necessitated Categoricalism (its supporters are the NCers). NC is supposed to be dissimilar from PP, since only the first accepts the existence of categorical properties; but according to neither position there are modal quiddities; the characterization of categoricity based on Modal Quidditism, while commonplace, is not suited to reconstruct the dissimilarity between NC and PP. Thus, it is not divisive.

Now, for dispositionality. The characterization of dispositionality according to which dispositional properties are necessarily associated with their dispositional profile may very well be comprehensive (it is applicable, as we have seen, to both pure powers and powerful qualities), but it is not divisive. The reason is quite straightforward: NC has properties necessarily associated with their dispositional profiles; so, as above, the properties of NC are dispositional. $\mathrm{NC}$ is supposed to be dissimilar from $\mathrm{PP}$ in matter of dispositionality, but, according to this characterization, it isn't. 
Summarizing, we have seen that constructing dispositionality and categoricity through the lenses of Modal Quidditism, a known strategy amongst PPers, is unsatisfactory for a number of reasons. Firstly because the two characterizations are mutually exclusive; secondly, because the characterization of categoricity is neither comprehensive nor divisive; thirdly, because the characterization of dispositionality is not divisive. The takeaway lesson from this subsection is that dispositionality and categoricity shouldn't be framed through the necessity or contingency of dispositional profiles, and therefore we shouldn't envision a modal divide as in Bird (2007a: pp. 66-67).

\subsection{PP and identity-based quidditism}

Identity-Based Quidditism is also a prominent candidate for categoricity (e.g., Bird 2007a: p. 44). Discussing what he calls "Humean" and "semi-Humean" views on properties and laws, Bird (2007a: p. 3) claims that.

there is very little on these views to the nature of a given property and certainly nothing that would distinguish it from some other property. The identity and distinctness of properties is a brute fact, not grounded in qualitative differences. Such a view of the nature of properties is called quidditism.

He then adds, shortly after, that:

[p]roperties whose natures are described by quidditism are traditionally known as categorical properties.

Bird is not the only one to think along these lines; according to Barker (2009: p. 242):

[a] property possesses a quiddity just in case its identity is fixed by something independent of the causal-nomological roles it may enter into. Paradigmatically, a categorical property is thought of as a property whose identity is fixed by a quiddity.

For the PPer, the characterization of categoricity via Identity-Based Quidditism goes hand in hand with a correspondent identity-based characterizations of dispositionality, according to which dispositional properties, unlike categorical ones, get their identity fixed through their dispositional profile, at least in the sense that properties are individuated by one of more pairs of stimulus/manifestation pairs to which the property is suitably related; as in Mumford (2004: p. 171), a property's identity “is fixed by relations with other properties". In this sense, PP is a close relative of the anti-quiddistic position according to which a property's identity is given by its unique position in a causal or nomic structure (e.g., Hawthorne 2001).

As in the case of Identity-Based Quidditism (Sect. 4.1), this claim concerning the identity and individuation of properties quickly turns into a claim about their nature, or essence: that properties have their identity fixed by their dispositional profiles is sometimes accompanied by the claim that properties are essentially dispositional, whereas categorical properties are not. There are various accounts concerning the 
dispositional essence of properties. ${ }^{10}$ What they all have in common, and what matters for our current purposes, is that dispositional essences make for necessary dispositional profiles, thus ensuring that the property's identity is fixed. This essentialist component of PP is so deep-rooted that Yates (2017: p. 4525) straightforwardly presents it as the position according to which "basic physical properties have wholly dispositional essences".

Thus, Identity-Based Quidditism is deployed by PPers as a characterization of categoricity as opposed to properties with a dispositional essence, whose identity is fixed relationally, through their dispositional profiles. In the simple words of Bird (2007a: p. 44):

[e]ssentially dispositional properties have their identities fixed by their dispositional characters; categorical properties have primitive identity.

Similarly, in the theory of properties put forward in Mumford (2004: p. 185):

[i]nstead of quiddities, the essence and identity of a property are determined by its relations to other properties.

Now we have identity-based characterizations of both dispositionality and categoricity. Are these useful for our purposes? Given that Identity-Based Quidditism does not entail Modal Quidditism, none of the problems of Modal Quidditism described above apply: most importantly, Identity-Based Quidditism, by allowing for categorical properties which are not modal quiddities, does not miscategorize PQ and NC, and allows the NCer to join the ranks of the Categoricalists.

That said, these identity-based characterizations may suffer from their own problems. Are they comprehensive and divisive? The short answer is: it depends on whether the PQer can accept them. Both of them are clearly acceptable by the PPer who submits them, and they are also acceptable by the Categoricalist in general (and by the NCer in particular), thus making the disagreement between the PPer and the Categoricalist a disagreement about the identity of properties (rather than a modal divide). But whether the similarities and dissimilarities concerning PQ are correctly reconstructed by this approach, depends on whether the PQer's properties are both dispositional and categorical according to these characterizations. This is what we now turn to.

\section{$5 \mathrm{PQ}$ and the identity-based characterizations}

PQers rarely claim that powerful qualities are powerful insofar as they have their identity fixed dispositionally_nor do they usually claim that properties have a dispositional essence. ${ }^{11}$ Concerning categoricity, perhaps the only PQer to explicitly state that powerful qualities are quiddities is Jacobs (2011) — and even then it is not clear

\footnotetext{
10 According to Bird (2007a), dispositional essences are to be cashed out in terms of subjunctive conditionals. According to Yates (2013), they are a primitive affair.

11 There are some noticeable, albeit fleeting, exceptions; according Martin and Heil (1999: p. 46), and Martin (2008: p. 45) powerful qualities do have a "dual nature".
} 
that the two characterizations match. ${ }^{12}$ It is therefore an active prescriptive effort to ascribe such identity-based characterizations to the PQer. But is it a viable route? This is a tricky subject that, I believe, has yet to be explored.

Right off the bat, we can see that -when it comes to categoricity-, Qualitative Identity and Identity-Based Quidditism perform very differently. Qualitative Identity (as in Sect. 4.1) is by definition incompatible with properties whose identity are fixed dispositionally -whereas the characterization of a property as a quiddity (viz. property with a primitive identity) prima facie says nothing about its dispositionality. ${ }^{13}$ So we might want to pursue this road: given that Identity-Based Quidditism does not, per se, define a property as non-dispositional, and given that there can be Identity-Based Quidditism without Modal Quidditism, it could be in principle possible to claim that powerful qualities are categorical to the extent that they have a primitive identity.

Could the PQer also adopt an identity-based characterization of dispositionality? As an indication, Bird (2007a: p. 44, fn. 38) claims that.

if one chooses to define a categorical property positively, as one which has a quiddity, then in principle a categorical property may have dispositional essence.

This leads to a position which Hawthorne (2001: p. 362) and Bird (2007a: p. 72, fn. 72) call the Double Aspect view (for brevity, DA): while properties have a dispositional essence, they also have a primitive quiddistic nature such that two dispositional properties may necessarily share the same dispositional profile. Given these characterizations of dispositionality and categoricity, DA qualifies as a position in the Mixed Family, as it posits properties that are both dispositional and categorical.

Can the PQer embrace DA, and thus be recognized by their PPer adversary? This is where we should take heed of the internal disagreements amongst PQers. The originators of the position proposed PQ as an Identity Theory, not simply in the sense that each dispositional property is identical to a categorical property (and vice versa), but in the stronger sense that, for each property, its dispositionality is identical to its categoricity, in turn identical to the property itself. ${ }^{14}$ This is what we will call $\mathrm{PQ}^{\text {id }}$ (and its supporters, $\mathrm{PQ}^{\text {id }}$ ers). Other PQers suspect this identity to be too robust to be properly defended, and settle for weaker positions, according to which powerful qualities are both dispositional and categorical by having distinct parts, or sides, or

\footnotetext{
12 Jacobs (2011: p.90) submits that categorical properties are "thick quiddities" in the sense that they "differ from each other, not merely numerically, but by nature"; however, as noted in Taylor (2018: p. 1427) this characterization of categorical properties as thick quiddities is not very divisive, as the PPer will surely agree that properties have their own nature; yet pure powers are not considered quiddities, as this nature is not primitive, but in turn depends on the relation(s) with stimulus and/or manifestation properties. Therefore, Jacobs (2011) and the PPers are not really in agreement by describing categorical properties as quiddities, thick or otherwise: the PPer is arguably pointing to a metaphysically more robust characterization.

13 Problems ensue if quidditistic properties are explicitly described as non-dispositional, a tempting characterization even for supporters of PQ: see the criticism of Schroer (2013) in Taylor (2013: p. 94). But a similar mistake is later repeated when Taylor (2018:p. 1437) deploys Lewis' (2009) notion of quiddities as "non-dispositional features of a property that primitively fix the identity of properties". Taylor (2018: p. 1437) is then quick to conclude that categorical properties cannot be dispositional.

14 Martin\&Heil (1999), Martin (1997, 2008), and Heil (2003). For an explicit formulation of the Identity Theory, Heil (2003: 111). For a recent and critical discussion, Giannotti (Forthcoming).
} 
aspects: we will call this subfamily of positions $\mathrm{PQ}^{\mathrm{da}}$ (and its supporters, $\mathrm{PQ}^{\mathrm{da}} \mathrm{ers}$ ). ${ }^{15}$ We can immediately point out that $\mathrm{PQ}^{\mathrm{da}}$ is better suited than $\mathrm{PQ}^{\mathrm{id}}$ to be framed along these identity-based characterizations of dispositionality, thus being equated with the DA position constructed by Bird and Hawthorne-whereas PQ qua Identity Theory is blocked from this conclusion.

That said, there is another difficulty plaguing the application of the identity-based characterizations to PQ, in all variants: suppose, for the sake of the argument, that all above difficulties are resolved and that a powerful qualities is both dispositional and categorical to the extent that its identity can be fixed both dispositionality and categorically. Now the problem, loosely speaking, is that the same job is being done twice, which results in a sort of overdetermination; the job is the "identity-fixing" of the property, performed for the property qua dispositional (through its dispositional profile), and for the property qua categorical (primitively). Not every overdetermination in metaphysics is vicious, and of course this also depends on what kind of operation the "identity-fixing" of a property is taken to be: yet this strikes as a further difficulty for the PQer. Contessa (2019: p. 13) may have been thinking along similar lines when discussing a problem of "explanatory profligacy" which, for him, plagues PQ:

[t]he supporter of POWERFUL QUALITIES [...] would seem to have not one but two explanations of what makes a certain universal the universal it is -i. e. each universal is both qualitatively distinguishable and dispositionally distinguishable from every other universal.

Here is a solution for this difficulty. One might claim that a property has its identity fixed partly dispositionally and partly categorically. Prima facie, that a property has its identity fixed partly dispositionally, might entail that the identity of a part of the property is fixed dispositionally (and another primitively), but this would entail that this solution is only available to the PQers who accept distinct parts of a property (a proper subset of $\mathrm{PQ}^{\mathrm{da}} \mathrm{ers}$, incidentally). However, this may not be the only way to understand this solution, as partly-fixing needn't be understood as the fixing of a part. This alternative understanding of the solution may be preferable for two reasons. First: it may be open to $\mathrm{PQ}^{\mathrm{id}} \mathrm{ers}$, as well as $\mathrm{PQ}^{\mathrm{da}} \mathrm{ers}$, as it doesn't require any internal complexity of the property to function. Secondly, it may rehabilitate the more general Qualitative Identity for the PQer (of any stripe): a powerful quality may have its identity partly fixed dispositionally, and partly in another way-whether primitively or not.

That said, I take it to be a highly contentious topic whether something can have its identity partially fixed, without having a part whose identity is fixed. For comparison, consider that by the standard logic of grounding, the fact $[\mathrm{P}(\mathrm{a})]$ partially grounds the fact $[\forall \mathrm{xP}(\mathrm{x})]$, although there doesn't seem to be any proper part of $[\forall \mathrm{xP}(\mathrm{x})]$ that $[\mathrm{P}(\mathrm{a})]$ might ground; that said, on certain assumptions $[\mathrm{P}(\mathrm{a})]$ may be said to partially ground $[\forall x P(x)]$ by being a part of it, much in the same way [p] is a partial ground of [p and q]

\footnotetext{
15 Martin (1993) was perhaps the original PQ ${ }^{\text {da }}$ er. More recently, Taylor (2018), Williams (2019), and Giannotti (Forthcoming).
} 
by being a part of it. That would still require that whatever is being partially grounded has parts.

In conclusion, the adoption of the PPer's identity-based characterizations is problematic for PQers of all stripes -although it would appear that $P Q^{\text {id }}$ ers have drawn the shortest end of the stick. The difficulties of course vary from position to position, and they needn't be unsurmountable, although they do pose their hurdles. ${ }^{16}$

In the following Section I will argue that there is a far less problematic route available, which involves a very peculiar solution: keeping only of the two identitybased characterizations, and discarding the other. This will eventually lead to my prescription.

\section{Prescribing a remedy}

\subsection{Discussion so far, and prospects for a solution}

Let us summarize the results achieved so far. Many of the PQer's characterizations of dispositionality and categoricity are non-divisive, thus unsuitable to reconstruct the debate between the PQer and its adversaries. On the other hand, the PPer's characterizations are mostly obtained with the help of quidditism. An oscillation has been noted between Modal Quidditism and Identity-Based Quidditism, and Modal Quidditism has been deemed unsuited for the job-thus showing that the dispositional/categorical divide is not a modal one as in Bird (2007a). Identity-based characterizations are indeed preferable, but they are still problematic if they want to be accepted by the PQer. Although the PQ ${ }^{\text {id }}$ er and the PQ ${ }^{\mathrm{da}}$ er may not entirely be on the same boat in this respect, there are some troubles to overcome if two different, and possibly conflicting stories are provided as to how one and the same property gets its identity fixed. To this day, PQers still have to rise to the challenge.

A less contentious prescription may thus be preferable. The general idea is to separate the identity-based characterization of categoricity from that of dispositionality, and to use the former without the latter. This has the immediate effect of solving the problems for PQ discussed in the previous Section, as no two conflicting stories are given as to how the property gets its identity fixed, and thus, no overdetermination arises. However, this strategy raises many questions: why would it be desirable to keep the identity-based characterization of categoricity, instead of dispositionality? And what should the latter be substituted with?

To answer such questions, note that the PQer, albeit open to the identity-based characterization of categoricity (as seen above), is not so open to an identity-based characterization of dispositionality - thus generating an initial asymmetry between the two, and a reason to keep one while rejecting the other. Here is why: as I have claimed, that a property has its identity fixed dispositionally means that its identity is fixed relationally, via a proper stimulus-manifestation relation. This makes the dispositional nature of a property explicitly relational, as in Bird (2007b); although sometimes, the

\footnotetext{
${ }^{16}$ E.g., can something partly fix the identity of a property, without fixing the identity of a part of the property? Can something really have its identity fixed primitively -but only in part? These are only some of the questions to arise in this scenario.
} 
slightly weaker claim is put forward that dispositional properties have a "directional" nature, as they essentially or constitutively point to their manifestations (Armstrong 1997: p. 69, Molnar 2003: p. 60).

This (pseudo-)relational characterization of dispositionality qua directedness, somewhat common amongst Categoricalists and PPers, has a problematic relation with PQ. This is straightforwardly clear for $\mathrm{PQ}^{\text {id }}$, given that Martin's (1997: p. 216) "surprising identity" between a property's dispositionality and the property itself makes it that no relational understanding of a property, or its nature, is allowed under its banner: dispositional properties are not relations (Heil 2003: p. 83; Martin 2008: p. 6); and even more specifically, as in Martin (1997: p. 216), "dispositionality is not a relation between what is dispositional and what is its manifestation".

Now, what about $\mathrm{PQ}^{\mathrm{da}}$ ? If a property is allowed to have distinct aspects or sides or parts, it may be less problematic to claim that one of them is relational, or at least directional in the way described by the PPer. But again, different positions might lead to different results: for one, it seems particularly pernicious to me to claim that a monadic (and possibly intrinsic) property has a relational part; what is more, directionality was originally understood by PPers as second-order relation (most clearly, Bird 2007b): yet how can a first-order monadic property has a second-order relation as a part? ${ }^{17}$ In general, given that directedness, and identity-based dispositionality, is not the go-to tool of the PQer, major surgery is required to retrofit the position, a surgery whose results are very much unclear. On the contrary, identity-based categoricity, in one of the variants proposed, does not require any adjustment to be applied. The only problems it displayed (e.g., the negative formulation of Qualitative Identity, or the overdetermination problem of Identity-Based Quidditism) stemmed by its being paired with a correspondent identity-based characterizations of dispositionality. Such considerations give us a reason to take the former while rejecting the latter.

\subsection{My prescription (and its virtues)}

I am finally in a position to present my proposal and its virtues, in order to correctly reconstruct similarities and dissimilarities between PP, PQ and Categoricalism—and thus to reconstruct this fragment of logical space.

Categoricity should be understood along the lines of an identity-based characterization, without being paired by any correspondent identity-based characterization of dispositionality. We have seen two such characterizations: Identity-Based Quidditism (properties have a primitive identity) and Qualitative Identity (properties do not have their identity fixed by any dispositional profile, but in some other way-whether primitively or not). Qualitative Identity is weaker than Identity-Based Quidditism, in the sense that it does not entail it but is entailed by it-and thus should be preferable. I do not want to dictate whether categorical properties have primitive identities; they might, or might not. Their identities, however, are not fixed by their dispositional profile.

This does not entail that categorical properties are not dispositional—as I reject the correspondent identity-based characterization of dispositionality: properties are not

\footnotetext{
17 Once again, less blunt versions of $\mathrm{PQ}^{\mathrm{da}} \mathrm{er}$-which do not explicitly deal in properties as having distinct parts or components-, may be less imperiled by the difficulty.
} 
dispositional by having their identities fixed in such-and-such a manner, whatever that might be. What is, then, dispositionality?

One apparently viable option that still has to be discussed is Privileged Contributory Dispositionality (as in Sect. 3.2), according to which a property is dispositional to the extent that it exclusively or essentially contributes to its bearer's being so-andso disposed. Some versions of Privileged Contributory Dispositionality are bound to present some familiar problems: viz. if we were to claim that a property contributes to its bearer's dispositionality by virtue of its (dispositional) essence, talk of a dispositional component to its nature or essence is bound to bounce back.

Thus, I suggest we understand Privileged Contributory Dispositionality in a different manner, not as an essential element of the property, but an explanatory one. ${ }^{18}$ Thus, I claim that a property is dispositional if and only if it is solely in virtue of possessing that property that its bearer is assigned a certain dispositional profile. We may cash this "in virtue of" locution in terms of metaphysical grounding, or an otherwise non-causal explanation; for our current purposes this is not terribly important. Three points however need pointing out:

(a) this explanatory/grounding relation needs to be metaphysically necessary. This ensures that dispositional properties are necessarily paired with their dispositional profiles; in the case of properties which are both dispositional and categorical, this ensures that they obey Qualitative Identity without being subject to Modal Quidditism.

(b) this characterization of a property's dispositionality is not about its essence, nor is an identity-based characterization. A property is not dispositional because it has a dispositional essence; dispositionality rather characterizes a property in relation to the explanatory/grounding roles it is capable of performing.

(c) This characterization of dispositionality works best in the context of the very first footnote of this paper, the one introducing the restriction to fundamental properties. Consider a macro-feature, e.g. fragility. There's no single property uniquely responsible for something being disposed to break if struck: it is rather a complex interplay of factors, properties and relations of parts, etc.... Yet this only means that it is unlikely that we will ever find a genuinely dispositional fundamental property of fragility - as one would expect. According to the friend of powers who accepts my characterization of dispositionality, certain other behaviors, presumably in privileged contexts, are due to unique (and fundamental) properties; although which ones, cannot be decided a priori by the metaphysician. In a second moment, we can verify how these dispositional and fundamental features are ultimately responsible for macro-features such as fragility (as the friends of powers occasionally say: no powers in, no powers out).

Finally, we can summarize my prescription as follows:

Categoricity as Qualitative Identity A property is categorical if and only if it does not have its identity fixed by any dispositional or nomic profile, but in some other way-whether primitively or not.

\footnotetext{
18 As perhaps implicitly in Heil (2003: p. 79), and more explicitly in Azzano (2019). An ampler selection of grounding-based theories of powers are more recently articulated in Tugby (2020).
} 
Dispositionality as Exclusive Contribution A property is dispositional if and only if it is solely in virtue of possessing that property that its bearer is assigned a certain dispositional profile.

So, how does this proposal perform? We notice right off the bat that the two characterizations are not mutually exclusive, and they do not require two ways of individuating a property, thus avoiding the overdetermination problem.

Then, of course, we must evaluate comprehensiveness and divisiveness. My characterization of dispositionality is comprehensive and divisive, for it can be applied to pure powers and powerful qualities alike, but not to the properties of the Categoricalist. Although this is not a claim they usually put forward, PPers can surely agree that instances of pure powers are all it takes to confer an object a certain dispositional profile (to be sure, a PPer would probably say something more, about the essence, identity and relatedness of properties, but they would agree with a PQer on this minimal point). Contrariwise, a Categoricalist takes properties to be just a part of the story of how an object is empowered; something else, say, a law of nature, or a primitive subjunctive fact, has to come to their help to endow objects with dispositions.

My characterization of categoricity as Qualitative Identity is similarly virtuous. It doesn't simply consider categorical properties as properties "with their own nature" (Taylor 2018: p. 1427), which would make it non-divisive; it also does not qualify categorical properties as non-dispositional quiddities (Taylor 2018: p. 1437), which would make the characterization unacceptable for the PQer. On the contrary, Qualitative Identity is both comprehensive and divisive. Let us see why.

It is claimed by Categoricalists that their properties have a primitive identity, and the claim is denied by PPers, thus making for a genuine dissimilarity between the two positions. Because Identity-Based Quidditism entails Qualitative Identity, then the Categoricalists claim, while the PPers deny, that properties obey Qualitative Identity. So far, so good. Now, as we have seen it is difficult to find PQers who explicitly cast categoricity based on how properties get their identity fixed; however, once the "rival" identity-based characterization of dispositionality is out of the picture, the proposal is viable. What is more, it may not only be viable, but also desirable. Here is why: the opposition between PP and PQ, at the end of the day, may be understood as the opposition between properties with a directional and relational nature, and traditional items more in line with Armstrong's (1997: p. 80) properties as "self-contained things, keeping themselves to themselves, not pointing beyond themselves to further effects brought about in virtue of such properties". To explicitly claim that powerful qualities obey Qualitative Identity might help to drive the point further, insofar as that would negate any need of a relational or directional element to fix a property's identity.

In conclusion, PP and PQ are similar with respect to dispositionality because they both have properties which are solely responsible for their bearers being so-and-so disposed, while this is not the case for Categoricalism; this ensures genuine agreement between PPers and PQers on matters of dispositionality, and genuine disagreement between them and the Categoricalist. PQ and Categoricalism are similar with respect to categoricity because they both have properties who obey Qualitative Identity, while this is not the case for PP (who contrariwise will go on to claim that properties have their identity fixed relationally or in an otherwise dispositional fashion). This ensures 
genuine agreement between PQer and Categoricalist on matters of categoricity, and genuine disagreement between them and the PPer.

The properly prescriptive element of the proposal is the following: the PQer should fully embrace the above characterization of dispositionality, which, as we have seen, was tentatively put forward by some PQer; and they should actively endorse Qualitative Identity, which is a viable and inviting proposition for them anyway. ${ }^{19}$ The PPer should endorse my characterization of dispositionality, which constitutes a viable proposition for them; the PPer is of course free to claim that properties have a dispositional essence, but this should not constitute their characterization of dispositionality itself, ensuring their agreement on the dispositionality of properties with the PQer. To claim that there are dispositional properties and to claim that properties have a dispositional essence, are two very different claims.

As a corollary, to be a friend of powers is not necessarily to accept properties with a dispositional essence, although often in the literature matters of dispositionality are loosely equated with matters of nature. ${ }^{20}$

\section{Additional features, objections, and directions for further research}

Finally, there are some features of (and objections to) my prescription that are worth discussing — some of which may provide future directions of research for friends of powers.

\subsection{Dispositionally indistinguishable properties}

My characterization of dispositionality does not exclude that, for whatever reason, two different properties may be solely responsible for their respective bearers being similarly disposed: thus, there might be two dispositionally indistinguishable properties.

I take this to be a feature, rather than a bug. Some friends of powers will be opposed to the possibility of dispositionally indistinguishable properties, and may deploy further metaphysical claims to motivate such an exclusion; e.g., a $\mathrm{PQ}^{\mathrm{id}} \mathrm{er}$ might claim that, by virtue of the Identity Theory each property is identical to its dispositionality; and a PPer might claim that, by virtue of a robust enough dispositional essentialism, a property's nature is entirely exhausted by its essential dispositions. Both solutions would exclude dispositionally indistinguishable properties from the get-go.

Other friends of powers might think differently, e.g. a $\mathrm{PQ}^{\mathrm{da}} \mathrm{er}$ might think that two properties can be dispositionally indistinguishable, yet have different categorical parts or aspects to differentiate what would otherwise be the same property. And, in my opinion, it doesn't stop there! Friends of powers need not resort to property-parts for this goal: perhaps two simple properties may be distinct, albeit dispositionally

\footnotetext{
19 A similar characterization of dispositionality and categoricity within the context of PQ has been very recently put forward in Coates (Forthcoming). Although the reasoning in that paper is somewhat different from mine, it shows a genuine philosophical pull in the same direction.

20 Yates (2017: p. 4526), Williams (2019: pp. 56-57).
} 
indistinguishable (as they ground the same dispositional profiles), as they have two distinct qualitative source of identities, whether primitive or not. ${ }^{21}$

There's no one-size-fits-all stance on this matter: I wouldn't want the friend of powers to give up on dispositionally indistinguishable properties just by way of having dispositional properties in their ontology. However, the friend of powers who accepts my prescription can still safely exclude them by reinforcing their position in some other way (e.g., in the ways suggested above), if they so desire.

\subsection{Essentialism vindicated?}

I have claimed that dispositionality is not a matter of essence, although some friends of powers may indeed think of properties as possessing a dispositional essence, by rejecting Qualitative Identity (or Identity-Based Quidditism). This is why amongst PPers one will likely find supporters of properties with dispositional essences.

Nevertheless, one might wonder whether essentialism — or something in the proximity thereof-is an inevitable consequence of my characterization of dispositionality. How is it, one might ask, that having a property solely grounds or explains how an object is disposed to behave? Is this to be understood as a brute correlation? Or perhaps it obtains because of how the property is, or its nature?

It is well-understood at this point that essentialism may provide an answer to metagrounding questions. As in Wallner (Forthcoming: 16) the grounding fact that event $e$ contains people engaged in conference-conducive activities ( $\mathrm{C}$-activities) grounds the fact that $e$ is a conference, may itself be "grounded in the fact that it is essential to conferences to contain people engaged in C-activities." Something similar could be true here: that having a certain property grounds being so-and-so disposed may be grounded in the fact that it is essential for that property to dispose so-and-so. ${ }^{22}$

One may very well think that: in fact, I think this is an excellent solution for dispositional essentialists. Yet it is not the only solution available. Even assuming, for the sake of the discussion, that my characterization of dispositionality involves a grounding relation such that it makes sense to ask what grounds its instances, essentialism is not the only solution. ${ }^{23}$ Amongst many, here is one I am sympathetic to, which Dasgupta (2014) calls Brute Nomicism; the essentialist solution and Brute Nomicism are two variants of what Dasgupta (2014) call "connectivism", according to which grounding facts are grounded with the help of a special connection holding between the two sides (e.g., between activities and conferences, or properties and dispositions). According to Brute Nomicism, however, this connection takes the form of a brute metaphysical principle, something akin to a "metaphysical law": e.g. that if something has a certain property, then it is so-and-so disposed; this law or principle is what guides the expla-

\footnotetext{
21 One may question how can these properties be numerically distinct if they have no distinct parts, as that would violate some basic extensionality principle. It seems to me that the mereology of properties hardly obeys such principles to begin with; e.g., nobody would object that logically simple (e.g., nonconjunctive) universals can be numerically distinct, on the ground that they do not have distinct parts. For the non-extensionality of second-order mereology, see Azzano (Forthcoming).

22 Or perhaps, as in McDaniel (2017: p. 231), it may be claimed that grounding is an internal relation, and, as such, grounded at least partially in the internal natures of its relata.

23 For an up-to-date summary of such other solutions, see Litland (2017) and Wallner (Forthcoming).
} 
nation from the property to the disposition. In other words, the introduction of certain entities in a systematic metaphysics (e.g., properties for the friend of powers) is connected to the postulation of certain grounding or explanatory axioms articulating how such entities are supposed to interact with the rest of the metaphysical architecture. To avoid an infinite regress of grounding facts, one may thus conclude (borrowing a famous expression from Schaffer 2016) that "it is the business of properties to dispose" and that would be the end of the story. That may be brute, but not less brute than the essentialist solution, which has to argue that it is essential of properties that they dispose, and that is the end of the story. After all, the essentialist still has to answer the question "why is it essential for properties to dispose?", which is why Dasgupta (2014) introduces Brute Nomicism back to back with what he calls Brute Essentialism. Brute Nomicism is intended as an alternative primarily for prospective friends of powers who do not envision dispositional essences, and believe that the explanatory association between powers and properties should be amenable to a treatment in terms of more familiar meta-philosophical items.

There's much that these positions have to clarify in this fascinating and hitherto unexplored intersection between power metaphysics and metametaphysics. Yet there's little to be gained, and much to be lost, by ignoring all non-essentialism-based approaches. $^{24}$

\subsection{About the identity theory}

My prescription wouldn't be so virtuous if some friend of powers couldn't accept it. Yet this could be the case of the PQ ${ }^{\text {id }}$ er. As already suggested, there's no univocal way to understand PQ qua Identity Theory between the dispositional and the categorical. In its strongest possible interpretation, the Identity Theory claims that there is no real difference between the property being dispositional, and the property being categorical. Yet, according to my characterizations, dispositionality and categoricity are two very different things. What should we do?

The most important thing to notice here is that such a robust understanding of the Identity Theory risks deflating PQ into a metaphysically more neutral view, such as NM; if there is no "oomphy" enough distinction between a property being dispositional and it being categorical, then any two distinct characterizations should be understood in a metaphysically deflated fashion, viz. as two different descriptions or ways of conceptualizing that property. $\mathrm{PQ}^{\mathrm{id}}$ ers themselves have flirted with this idea, at least since Martin's (1997: p. 216) notorious suggestion that one should think about dispositionality and categoricity like the faces and the goblet in the Rubin's Vase ambiguous drawing. That said, as claimed in the beginning of Sect. 3, our current proposition is to investigate dispositionality and categoricity as genuine metaphysical categories, and thus we must preserve the distinction between PQ and NM, or any similarly deflated understanding of the position. Given what has been just said, however, this may bear the unfortunate consequence of making $\mathrm{PQ}^{\mathrm{id}}$, in its possible strongest

\footnotetext{
24 Perhaps, grounding itself may collapse unto essence (Correia 2013), or both might reduce to a third notion, e.g. generalized identity (Correia and Skiles 2017). The metaphysical rebooting needed to discuss such options, I take it, goes well beyond the scope of this paper.
} 
interpretation, a problematic and unstable position. This has little to do, mind you, with my prescription: the same problem would have presented itself with any two distinct characterizations of dispositionality and categoricity.

But there are good news: I believe that a virtue of my proposal is that it might allow the PQer to endorse a version of Identity Theory stronger than most variants of $\mathrm{PQ}^{\mathrm{da}}$. Once again, it is the divorce between identity-based categoricity from the identity-based dispositionality to do the job: loosely put, the PQ ${ }^{\text {id }}$ er's problem was that two such characterizations, when adopted together, were wresting against each other in a property that was supposed to display no internal complexity. This does not happen here. While categoricity as Qualitative Identity characterizes the property as one with a certain nature or identity, dispositionality as Exclusive Contribution merely characterizes it as taking part in certain explanatory patterns, and doesn't require it to have any particular composition, internal make-up, or nature of any kind.

Some versions of $\mathrm{PQ}^{\mathrm{da}}$, I suspect, may approximate these results. ${ }^{25}$ This may be taken to show that there's little wiggle room between a less problematic $\mathrm{PQ}^{\mathrm{id}}$ and $\mathrm{PQ}^{\mathrm{da}}$, or, alternatively, that the lines between the two are blurry. That may very well be, but my point is rather the following: if we do not want to deflate PQ into a metaphysically neutral view, the strongest interpretation of the Identity Theory is a very problematic and unstable position. My prescription allows one to endorse the next best thing.

\subsection{A distinction between categoricity and qualitativity}

In this paper, I have followed the quasi-orthodoxy of using "categorical" and "qualitative" almost interchangeably. PQers are known to claim that properties are qualitative, but also claim that they are categorical — at least, there's no clear distinction between the two.

Perhaps that was a mistake. For there is another, hitherto unexplored characterization of categoricity that might prise them apart: this is the characterization of a categorical property as an actual non-conditional and intrinsically inert feature of an object. ${ }^{26}$ This is a venerable characterization of a categorical property, which ties back to the distinction between neo-Humeans and anti-Humeans metaphysical settings. Of course, however, powerful qualities are not intrinsically inert, and as such they cannot be categorical in this sense-although they may very well be qualitative.

Two different conclusions can be reached from this fact. Conclusion (1)-because powerful qualities are not intrinsically inert, and because supposedly there are categorical properties according to $\mathrm{PQ}$, then this characterization of categoricity is unsatisfactory. This is but an instance of my method in Sect. 2: the PPer and the PQer do not disagree as to whether properties are intrinsically inert, but they supposedly disagree as to whether properties are categorical. Thus, the "intrinsically inert" characterization is unsatisfactory, as venerable as it is: for it is non-divisive. However, in my account the notion of a property as intrinsically inert can be tied to its nondispositionality: roughly put, according to my prescription dispositionality amounts to the fact that having a property is all it takes to have a dispositional and nomic

\footnotetext{
25 E.g., Giannotti (Forthcoming).

${ }^{26}$ Black (2000: p. 91), Bird (2007a: p. 67).
} 
dimension, and perhaps a causal one as well. Objects, as long as they have certain properties, do not need anything else to kick themselves into action, and properties are primarily responsible for that activity. Foes of powers on the contrary believe properties to be insufficient for the job, because inherently non-modal and non-causal. Drawing Conclusion (1), my account in Sect. 6.2 now reads as follow: the PPer and the PQer agree that properties are dispositional (as satisfying Exclusive Contribution, and thus not intrinsically inert); while they both disagree with the Categoricalist on this matter. The PQer and the Categoricalist agree that properties are categorical, or qualitative (as satisfying Qualitative Identity); while they both disagree with the PPer on this matter.

The opposing Conclusion (2) is different. Let us assume that, going into the paper, we have a clear enough grip on the notion of categoricity even before deploying my method by comparison, such that we can safely claim that categorical properties are "actual, non-conditional, and intrinsically inert" features of an object. Thus, the PQer shouldn't really claim that properties are categorical (although they are qualitative). More generally, on matters of categoricity, the PQer doesn't really disagree with the PPer and agree with the Categoricalist, as it would prima facie appear. The other way round is true! If Conclusion (2) is drawn, then, my account is to be read as follows: the PPer and the PQer agree that properties are dispositional (as satisfying Exclusive Contribution), and they also should agree that they are not categorical (as not intrinsically inert); while they both disagree with the Categoricalist on both matters. The PQer and the Categoricalist agree that properties are qualitative (as satisfying Qualitative Identity); while they both disagree with the PPer on this matter.

These two prescriptions are very similar, but with one distinction: the former links categoricity with qualitativity, whereas the latter links it with non-dispositionality.

So, which one is it? That depends, amongst other things, on whether we can safely assume that categoricity is tied to the "intrinsically inert" characterization even before deploying my method-viz. by whether we can claim there to be a secure enough and independent grasp on the notion of categoricity. ${ }^{27}$

I do not wish to resolve this tension, viz. I will not choose whether categoricity should be associated with qualitativity (qua Qualitative Identity) or non-dispositionality (qua Exclusive Contribution, and thus, the "intrinsically inert" characterization). My prescription satisfactorily reconstructs similarities and differences in the background metaphysics between positions in this portion of the logical space. Which words we decide to attach to them is, I suspect, a secondary matter.

\section{Conclusion}

I offered a clearer terrain of engagement between PPers, PQers, and Categoricalists, by clarifying what it is that they should mean when they claim that properties are dispositional, categorical, or both. It has not been an easy task. Most of the characterizations offered by the PQer have been shown to be crucially non-divisive, while the

\footnotetext{
27 One may prefer Conclusion 1 simply because it does not force one to aggressively debate PQers on the correct use of the expression "categorical". This is, to my mind, a perfectly acceptable stance.
} 
modal characterizations employed by the PPer, which often lead them to believe the dispositional/categorical divide to be a modal one (as in Bird 2007a), are both noncomprehensive and non-divisive. All in all, such characterizations fail to correctly carve at the joints of the debate. Additionally, the identity-based characterizations of the PPer interact problematically with PQ.

My prescription functions on an original premise: isolating Qualitative Identity as a successful identity-based characterization of categoricity, while abandoning the correspondent identity-based characterization of dispositionality. In turn, dispositionality should be cashed out in other terms: a property is dispositional if and only if it is solely responsible for its bearer having a certain dispositional profile. The main point of contention between PPers and PQers - as to whether properties are categorical as well as dispositional-is to be understood as whether properties, responsible for their bearers being so-and-so disposed, also have a nature or identity fixed by a stimulus and manifestation properties in a (pseudo-)relational fashion. Finally, the most crucial consequence of the prescription is that a property is not necessarily dispositional by having a certain nature or essence; and while PPers claim that dispositional properties have a dispositional essence, this needn't be a claim endorsed by all friends of powers.

As demonstrated by the last Section, there are many issues still on the table: can two properties be dispositionally indistinguishable? How robust can the Identity Theory be? Is there a difference between categoricity and qualitativity? That said, my prescription has been proven successful by the criteria set-up in Sect. 2, and has managed to clear the air in a limited (yet populated) fragment of the logical space of positions concerning dispositionality and categoricity—ultimately providing a solid terrain of engagement for future discussions.

Acknowledgements I presented an early draft of this paper at the New Foundations of Dispositionalism conference at the University of Exeter (May 2019); I would like to thank the audience for their comments and discussions on these topics. I would also like to thank two anonymous reviewers for their particularly insightful comments.

Funding Open access funding provided by Università degli Studi di Bergamo within the CRUI-CARE Agreement.

Open Access This article is licensed under a Creative Commons Attribution 4.0 International License, which permits use, sharing, adaptation, distribution and reproduction in any medium or format, as long as you give appropriate credit to the original author(s) and the source, provide a link to the Creative Commons licence, and indicate if changes were made. The images or other third party material in this article are included in the article's Creative Commons licence, unless indicated otherwise in a credit line to the material. If material is not included in the article's Creative Commons licence and your intended use is not permitted by statutory regulation or exceeds the permitted use, you will need to obtain permission directly from the copyright holder. To view a copy of this licence, visit http://creativecommons.org/licenses/by/4.0/.

\section{References}

Armstrong, D. M. (1997). A world of states of affairs. Cambridge: Cambridge University Press.

Armstrong, D. M. (2004). How do particulars stand to universals? In D. Zimmerman (Ed.), Oxford studies in metaphysics (pp. 139-154). England: Oxford University Press.

Armstrong, D. M. (2005). Four disputes about properties. Synthese, 144(3), 309-320.

Azzano, L. (2019). The question of realism for powers. Synthese, 196(1), 329-354. 
Azzano, L. (Forthcoming). Structural properties, mereology, and modal magic. Synthese.

Barker, S. (2009). Dispositional monism, relational constitution and quiddities. Analysis, 69(2), $242-250$.

Borghini, A., \& Williams, N. (2008). A dispositional theory of possibility. Dialectica, 62(1), 21-41.

Bird, A. (2003). Structural properties. In G. Rodriguez-Pereyra \& H. Lillehammer (Eds.), Real metaphysics (pp. 155-168). Abingdon: Routledge.

Bird, A. (2007a). Nature's metaphysics: laws and properties. England: Oxford University Press.

Bird, A. (2007b). The regress of pure powers? Philosophical Quarterly, 57(229), 13-534.

Bird, A. (2009). Structural properties revisited. In T. Handfield (Ed.), Dispositions and Causes (pp. 215-241). UK: Clarendon Press.

Bird, A. (2016). Overpowering: how the powers ontology has overreached itself. Mind, 125(498), 341-383.

Black, R. (2000). Against quidditism. Australasian Journal of Philosophy, 78(1), 87-104.

Chisholm, R. M. (1967). Identity through possible worlds: Some questions. Nô̂s, 1(1), 1-8.

Coates, A. (Forthcoming). Making sense of powerful qualities. Synthese.

Contessa, G. (2019). Powerful qualities or pure powers? Metaphysica, 20(1), 5-33.

Correia, F. (2013). Metaphysical grounds and essence. In M. Hoeltje, B. Schniederm, \& A. Steinberg (Eds.), Varieties of dependence: ontological dependence, grounding, supervenience, response-dependence. Munich: Philosophia Verlag.

Correia, F., \& Skiles, A. (2017). Grounding, essence and identity. Philosophy and Phenomenological Research, 3, 642-670.

Dasgupta, S. (2014). The possibility of physicalism. Journal of Philosophy, 111(9-10), 557-592.

Ellis, B. (2001). Scientific essentialism. Cambridge: Cambridge University Press.

Ellis, B., \& Lierse, C. (1994). Dispositional essentialism. Australasian Journal of Philosophy, 72(1), $27-45$.

Engelhard, K. (2010). Categories and the ontology of powers: A vindication of the identity theory of properties. In A. Marmodoro (Ed.), The metaphysics of powers: Their grounding and their manifestations (pp. 41-57). Routledge.

Giannotti, J. (Forthcoming). The Identity Theory of Powers Revisited. Erkenntnis.

Hawthorne, J. (2001). Causal structuralism. Philosophical Perspectives, 15, 361-378.

Heil, J. (2003). From an ontological point of view. England: Oxford University Press.

Heil, J. (2005). Dispositions. Synthese, 144(3), 343-356.

Heil, J. (2012). The universe as we find it. England: Oxford University Press.

Ingthorsson, R. D. (2013). Properties: qualities, powers, or both? Dialectica, 67(1), 55-80.

Jacobs, J. (2011). Powerful qualities, not pure powers. The Monist, 94(1), 81-102.

Lewis, D. K. (1986). On the plurality of worlds. NJ: Wiley.

Lewis, D. K. (2009). Ramseyan humility. In D. Braddon-Mitchell \& R. Nolan (Eds.), Conceptual analysis and philosophical naturalism (pp. 203-222). MIT Press.

Litland, J. (2017). Grounding grounding oxford studies in metaphysics 10. England: Oxford University Press.

Locke, D. (2012). Quidditism without quiddities. Philosophical Studies, 160(3), 345-363.

Lowe, E. J. (2010). On the individuation of powers. In A. Marmodoro (Ed.), The metaphysics of powers: Their grounding and their manifestations (pp. 8-26). Routledge.

Martin, C. B. (1993). The need for ontology: some choices. Philosophy, 68(266), 505-522.

Martin, C. B. (1997). On the need for properties: The road to pythagoreanism and back. Synthese, 112(2), 193-231.

Martin, C. B. (2008). The mind in nature. England: Oxford University Press.

Martin, C. B., \& Heil, J. (1999). The ontological turn. Midwest Studies in Philosophy, 23(1), 34-60.

McDaniel, K. (2017). The fragmentation of being. England: Oxford University Press.

McKitrick, J. (2018). Dispositional pluralism. England: Oxford University Press.

Mellor, D. H. (1974). defense of dispositions. Philosophical Review, 83(2), 157-181.

Mellor, D. H. (1982). Counting corners correctly. Analysis, 42(2), 96-97.

Molnar, G. (2003). Powers: A study in metaphysics. England: Oxford University Press.

Mumford, S. (1998). Dispositions. England: Oxford Clarendon Press.

Mumford, S. (2004). Laws in nature. Abingdon: Routledge.

Nolan, D. (2009). Ramseyan humility. In D. Braddon-Mitchell \& R. Nolan (Eds.), Conceptual analysis and philosophical naturalism (pp. 203-222). Cambridge: MIT Press.

Prior, E. (1982). The dispositional/categorical distinction. Analysis, 42(2), 93-96.

Schaffer, J. (2016). It is the business of laws to govern. Dialectica, 70(4), 577-588.

Schroer, R. (2010). Is there more than one categorical property? Philosophical Quarterly, 60(241), 831-850. 
Schroer, R. (2013). Can a single property be both dispositional and categorical? Metaphysica, 14(1), 63-77. Shoemaker, S. (1980). Causality and properties. In P. van Inwagen (Ed.), Time and cause. D (pp. 109-35). Austria: Reidel.

Smith, D. (2016). Quid quidditism est? Erkenntnis, 81(2), 237-257.

Strawson, G. (2008). The identity of the categorical and the dispositional. Analysis, 68(300), 271-282.

Taylor, J. (2013). Defence of Powerful Qualities. Metaphysica, 14(1), 93-107.

Taylor, J. (2018). Powerful qualities and pure powers. Philosophical Studies, 175(6), 1423-1440.

Tugby, M. (Forthcoming). Grounding theories of powers. Synthese.

Wallner, M. (Forthcoming). The ground of ground, essence, and explanation. Synthese.

Williams, N. E. (2019). The powers metaphysic. Oxford University Press.

Yates, D. (2013). The essence of dispositional essentialism. Philosophy and Phenomenological Research, $87(1), 93-128$.

Yates, D. (2017). Inverse functionalism and the individuation of powers. Synthese, 195(10), 4525-4550.

Publisher's Note Springer Nature remains neutral with regard to jurisdictional claims in published maps and institutional affiliations. 